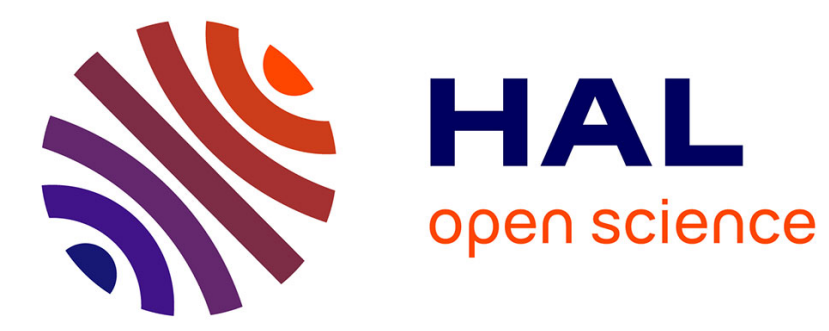

\title{
Phenomenological modeling of tumor diameter growth based on a mixed effects model
}

Thierry Bastogne, Adeline Samson, Pierre Vallois, Sophie Mézières-Wantz, Sophie Pinel, Denise Bechet, Muriel Barberi-Heyob

\section{- To cite this version:}

Thierry Bastogne, Adeline Samson, Pierre Vallois, Sophie Mézières-Wantz, Sophie Pinel, et al.. Phenomenological modeling of tumor diameter growth based on a mixed effects model. Journal of Theoretical Biology, 2010, 262 (3), pp.544-552. 10.1016/j.jtbi.2009.10.008 . hal-00390380

\section{HAL Id: hal-00390380 \\ https://hal.science/hal-00390380}

Submitted on 2 Jun 2009

HAL is a multi-disciplinary open access archive for the deposit and dissemination of scientific research documents, whether they are published or not. The documents may come from teaching and research institutions in France or abroad, or from public or private research centers.
L'archive ouverte pluridisciplinaire HAL, est destinée au dépôt et à la diffusion de documents scientifiques de niveau recherche, publiés ou non, émanant des établissements d'enseignement et de recherche français ou étrangers, des laboratoires publics ou privés. 


\section{Phenomenological modeling of tumor diameter growth based on a mixed effects model}

T. Bastogne ${ }^{\text {a,* }}$, A. Samson ${ }^{\text {c }}$, P. Vallois ${ }^{\mathrm{d}}$, S. Wantz-Mézières ${ }^{\mathrm{d}}$,

S. Pinel ${ }^{\mathrm{e}}$, D. Bechet ${ }^{\mathrm{b}}$, M. Barberi-Heyob ${ }^{\mathrm{b}}$.

${ }^{\mathrm{a}}$ Centre de Recherche en Automatique de Nancy (CRAN)

Nancy - Université, CNRS UMR 7039,

BP 239, F-54506 Vandœuvre-lès-Nancy Cedex, France,

Phone: (33) 383684473 - Fax: (33) 383684462

${ }^{\mathrm{b}}$ Centre de Recherche en Automatique de Nancy (CRAN)

Nancy - Université, CNRS UMR 7039,

Centre Alexis Vautrin, Centre de Lutte contre le Cancer

Brabois, Av. de Bourgogne, 54511 Vandouvre-lès-Nancy Cedex, France

${ }^{\mathrm{c}}$ Laboratoire MAP5

Université Paris Descartes, CNRS UMR 8145, Paris

d Institut de Mathématiques Elie Cartan,

Nancy-Université, CNRS UMR 7502,

BP 239, F-54506 Vandouvre-lès-Nancy Cedex, France

e Signalisation, Génomique et Recherche Transactionnelle en Oncologie,

Nancy-Université 


\begin{abstract}
Over the last few years, taking advantage of the linear growth of diameter kinetics, tumor diameter-based rather than tumor volume-based models have been developed for the phenomenological modeling of tumor growth. In this study, we propose a new tumor diameter growth function composed of two linear parts and one exponential term to characterize early, late and steady-state treatment effects. Model parameters consist of growth rates, growth delays and time constants and are meaningful for biologists. Biological experiments provide in vivo longitudinal data. The latter are analyzed using a mixed effects model based on the new diameter growth function, to take into account inter-mouse variability and treatment factors. The relevance of the tumor growth mixed model is firstly assessed by analyzing the effects of three therapeutic strategies for cancer treatment (radiotherapy, concomitant radiochemotherapy and photodynamic therapy) administered on mice. Then, effects of the radiochemotherapy treatment duration are estimated within the mixed model. The results highlight the model suitability for analyzing therapeutic efficiency, comparing treatment responses and optimizing, when used in combination with optimal experiment design, anti-cancer treatment modalities.
\end{abstract}

Key words: empirical model-building, tumor growth, mixed models, cancer

\footnotetext{
$\bar{*}$ Corresponding author.

Email address: thierry.bastogne@cran.uhp-nancy.fr (T. Bastogne).
} 


\section{Introduction}

In systems theory ${ }^{1,2}$, phenomenological or black-box models are holistic representations of complex systems in nature, society, and science. This study presents a new contribution of this modeling approach in oncology. Efficient developments of new therapeutic strategies for cancer treatment require a reliable, robust and reproducible evaluation of therapeutic effects. In in vivo growth inhibition studies, the most commonly used characteristics of tumor growth are tumor growth delay (TGD) and tumor volume $\mathrm{T} / \mathrm{C}$ value ${ }^{3}$. TGD and $\mathrm{T} / \mathrm{C}$ are both based on a local event, either the time instant associated with the quadrupling of the tumor volume or the relative tumor volume at a given time point after treatment respectively. Accordingly, these two characteristics only give quantitative information about the tumor growth at an event point but provide no information about the global behavior of the tumor after this event. To remedy this problem, a solution consists in building parametric models of tumor growth ${ }^{4-6}$. Those models have to be simple enough so that they can be analyzed with available mathematical techniques, and accurate enough to describe the important aspects of the relevant dynamical behavior. By 'relevance' we mean taking into consideration of three main issues raised by tumor growth modeling.

- Choice of the response variable. The choice of the response variable to be modeled is not obvious. In most usual tumor growth models, e.g. population dynamics models, compartmental models or cell-cycle models, the explained variable is the number of cancer cells in the tumor population ${ }^{7-14}$. However, the tumor size is not easily measurable in in vivo contexts. For this reason, in vivo tumor growth models are often based on the tumor volume that is supposed to be proportional to the number of cancer cells ${ }^{7,15}$. However, 
Drasdo et al. showed in ${ }^{16-18}$ that tumor growth kinetics follows a power-law growth for large times and suggested to use the tumor diameter as response variable because of its linear growth kinetics. The in vivo tumor diameter growth model proposed in this study is built on this assumption.

- Description of the inter-individual variability. The experiment design provides longitudinal data with few observation times but with repeated measurements among subjects. Indeed in vitro as well as in vivo experiments are always repeated to assess the reproducibility degree of the experimental responses. In classic regression approaches, the model parameters are supposed to be identical for all subjects. However, in experimental biology, the inter-individual variability makes this assumption inappropriate. Another approach consists in describing each model parameter as a sum of fixed and random effects. The so-called mixed effects models (or mixed models) allow taking into account this lack of response reproducibility. They have proved their efficiency, particularly in biomedical applications ${ }^{19-21}$.

- Identification of influent treatment modalities. A large majority of tumor growth models does not take into account input causes like treatment factors. As a consequence, those noncausal models are not suited to the modelbased control of anticancer treatments. Such relation of cause and effect can be estimated in mixed models by introducing covariate effects in the expression of model parameters ${ }^{19}$.

In this study, we propose to adopt the mixed model methodology to describe the tumor diameter growth. This suggested approach is carried out in four successive steps:

(1) Data collection. Experimental data are time series of tumor diameters, measured once every two days after a tumor implantation on nude mice, using an electronic caliper. Three different therapeutic strategies for can- 
cer treatment (radiotherapy, concomitant radiochemotherapy and photodynamic therapy) are administered to three mouse groups. In the radiochemotherapy mouse group, three treatment durations are compared.

(2) Choice of the model structure. We develop a model with two linear trends and one exponential part.

(3) Parameter estimation. The parameter estimation of the mixed model is performed with a Stochastic Approximation Expectation-Maximization (SAEM) algorithm developed by Kuhn and Lavielle in ${ }^{22}$.

(4) Analysis of treatment effects. Treatment group covariates are introduced in the mixed model and selected using statistical tests. Significant differences are emphasized between, on the one hand, therapeutic strategies and, on the other hand, treatment durations in radiochemotherapy.

This paper is organized as follows. In Section 2, a new empirical kinetic model of tumor growth is proposed. Experimental setup of data collection and statistical methods are then presented in Section 3. Modeling results are analyzed in Section 4. The case of non-treated tumor growth is firstly examined. In a second subsection three loco-regional therapies for cancer treatment are compared. Finally the estimation results for the concomitant radiochemotherapy group to assess effects of the treatment duration are presented. The conclusions and perspectives of this work are drawn in Section 5.

\section{Tumor growth modeling}

This Section first defines the response variable (tumor diameter). Next, we suggest a new model structure of tumor diameter growth. To identify this model from longitudinal data (see Figure 2(a)), a statistical mixed effects representation is then defined. The notations used in this study are listed in 
Table 1.

\subsection{Response variable}

In growth inhibition studies, the usual response variable is the tumor volume, $v(t)$. This quantity is defined at day $t$ as

$$
v(t)=\frac{\delta_{1}(t) \delta_{2}(t)^{2}}{2}
$$

where $\delta_{1}(t)$ and $\delta_{2}(t)$ are the long and short axis dimensions, respectively, of the ellipse formed by the tumor. $\delta_{1}(t)$ and $\delta_{2}(t)$ are measured every two days in two orthogonal directions using an electronic caliper. The mean tumor diameter could first be computed as $y(t)=\left(\delta_{1}(t)+\delta_{2}(t)\right) / 2$. Unfortunately, $\delta_{1}(t)$ and $\delta_{2}(t)$, contrary to $v(t)$, are seldom given by experimenters. That led us to define the response variable as the diameter of a fictitious spherical tumor of volume $v$,

$$
y(t)=\sqrt[3]{\frac{6 v(t)}{\pi}} .
$$

\subsection{Tumor growth model structure}

Treated and non-treated tumor growth responses are described by a LinearExponential-Linear (LEL) model structure in which $x(t)$ denotes the explained diameter of the tumor at time $t$,

$$
x(t)=x_{0}[\underbrace{1+a t}_{\begin{array}{c}
\text { natural } \\
\text { growth }
\end{array}}+\underbrace{\left(x_{1}(t)+x_{2}(t)+x_{3}(t)\right) u}_{\text {treatment response }}]
$$


Table 1

Main notations

\begin{tabular}{|c|c|c|}
\hline Symb. & Description & Unit \\
\hline$t$ & time & day \\
\hline$x(t)$ & model output (explained diameter of the tumor) & $m m$ \\
\hline$y(t)$ & measured response variable (tumor mean diameter) & $m m$ \\
\hline$t_{f}$ & time of the last observation, before sacrifice of the mouse & \\
\hline$c$ & treatment covariate & \\
\hline$d$ & number of model parameters & \\
\hline$r$ & number of repeated experiments (nb of mice / group) & \\
\hline$i$ & index of the subject (mouse) with $i \in\{1, \cdots, r\}$ & \\
\hline$n$ & number of observations & \\
\hline$n_{i}$ & number of observations for the $i^{t h}$ mouse & \\
\hline$j$ & $j^{\text {th }}$ observation with $j \in\{1, \cdots, n\}$ & \\
\hline$\Theta$ & vector of the model parameters in $\mathbb{R}^{d}$ & \\
\hline$\beta_{\theta, c}$ & effect of the covariate $c$ on the model parameter $\theta$ & \\
\hline$\Omega$ & covariance matrix of random effects & \\
\hline$p$ & number of covariates & \\
\hline$\sigma$ & variance of the within-group output error & \\
\hline$a$ & natural diameter growth rate of the tumor & $d a y^{-1}$ \\
\hline$b$ & decrease rate of tumor diameter in treatment phase I & $d a y^{-1}$ \\
\hline$k_{1}=b-a$ & resulting diameter growth rate in phase I & $d a y^{-1}$ \\
\hline$k_{2}$ & slope of diameter decrease at time $\tau$ in treatment phase II & \\
\hline$k_{3}$ & decrease rate of tumor diameter in treatment phase III & $d a y^{-1}$ \\
\hline$\tau$ & time delay of phases II and III & day \\
\hline$T$ & time constant of phase II & day \\
\hline$x_{0}$ & initial value of the tumor diameter & $m m$ \\
\hline PDT & photodynamic therapy & \\
\hline $\mathrm{RCT}$ & concomitant radiochemotherapy & \\
\hline $\mathrm{RT}$ & radiotherapy & \\
\hline
\end{tabular}


with:

$$
\begin{aligned}
& x_{1}(t)=-b t \\
& x_{2}(t)=-k_{2} T\left(1-e^{-(t-\tau) / T}\right) H(t-\tau) \\
& x_{3}(t)=-k_{3}(t-\tau) H(t-\tau),
\end{aligned}
$$

where $t \in\left[0 ; t_{f}\right] . t=0$ denotes the treatment beginning day and $t_{f}$ is either sacrifice day (day at which the tumor reaches a size limit of about $15 \mathrm{~mm}$ diameter) or cure day (day at which the tumor is no longer perceptible, $x(t) \leq$ $\varepsilon$ for $t \geq t_{f}, \varepsilon$ corresponding to the minimum measurable diameter by the caliper). $u$ denotes the treatment variable: $u=0$ for non-treated tumors and $u=1$ for treated tumors. $H(t)$ is the Heaviside step function and $x_{0}=x(0)$ is the unknown initial value of the tumor diameter. Given (3), the tumor growth rate $\rho(t)$ is defined by

$$
\rho(t)=\frac{x(t)-x_{0}}{x_{0}}=a t+\left(x_{1}(t)+x_{2}(t)+x_{3}(t)\right) u,
$$

and can be split up into four parts:

- the natural growth phase (at) where the parameter a denotes the mean growth rate of the tumor diameter over one day;

- the early treatment effect $\left(x_{1}(t)\right)$. A positive value of $b$ denotes a mean decrease rate of the tumor diameter over one day. $a$ and $b$ were gathered into a global rate coefficient $k_{1}=b-a$ to avoid identifiability problems for treated tumors;

- the late treatment effect $\left(x_{2}(t)\right)$ starting at time $\tau$, where $T$ is a time constant and $k_{2}=\partial x_{2} /\left.\partial t\right|_{t=\tau}$ corresponds to the slope of the tumor size decrease at time $\tau$. Those two parameters, $T$ and $\tau$, take into account the duration and magnitude of the late effect; 
- the steady-state effect $\left(x_{3}(t)\right)$. The latter corresponds to the post-treatment effect, where $k_{3}$ denotes a mean decrease rate of the tumor diameter over one day. At steady-state, the global growth rate is given by $a-b-k_{3}$. Note that $\tau$ both takes place in phases II and III.

As illustrated in Figure 1, kinetic effects associated with $x_{1}, x_{2}, x_{3}$ are superimposed to natural growth response to give the resultant treated growth kinetics. From now on, $\boldsymbol{\Theta}$ will denote the vector of parameters, i.e. $\boldsymbol{\Theta}=\left(x_{0}, a\right)$ for non-treated growth kinetics and

$$
\Theta=\left(x_{0}, k_{1}, k_{2}, T, \tau, k_{3}\right)
$$

for treated growth kinetics. These parameters have biological significance (initial size, diameter growth rates, time constant and time delay). Any positive enhancement of one parameter among $k_{1}, k_{2}, k_{3}, T$ and $\tau$ suggests a local therapeutic improvement during the corresponding phase of growth. Conversely, any decrease of one of the latter parameters leads to locally degrading the therapeutic response.

\subsection{Mixed effects model}

Let $y_{i j} \in \mathbb{R}$ denote the noisy measurement of tumor diameter for subject $i=1, \ldots, r$ at time $t_{i j}$ with $j=1, \ldots, n_{i}$ and $n_{i}$ the number of observations (time points) of subject $i$. In our case, the mixed model of the tumor growth is given by:

$$
y_{i j}=x\left(t_{i j}, \mathbf{\Theta}_{i}\right)+\epsilon_{i j}, \quad \forall i=1, \ldots, r, \forall j=1, \ldots, n_{i},
$$


where $x\left(t_{i j}, \Theta_{i}\right)$ is given by equation (3) and denotes the explained diameter of subject $i$ at time $t_{i j}$ depending on the individual parameter vector $\boldsymbol{\Theta}_{i}$ of length $d$. The within-group output error $\epsilon_{i j}$ is described either by a homoscedastic error model of the type

$$
\epsilon_{i j}=\sigma e_{i j}, e_{i j} \sim_{i . i . d .} \mathcal{N}(0,1), \quad \forall i=1, \ldots, r, \forall j=1, \ldots, n_{i},
$$

or a heteroscedastic error model

$$
\epsilon_{i j}=\sigma x\left(t_{i j}, \boldsymbol{\Theta}_{i}\right) e_{i j}, e_{i j} \sim_{i . i . d .} \mathcal{N}(0,1), \quad \forall i=1, \ldots, r, \forall j=1, \ldots, n_{i},
$$

where $\sigma$ is the unknown standard error.

To take into account the inter-individual variability, the individual parameter vectors $\left(\boldsymbol{\Theta}_{i}\right)$ are assumed to be mainly Gaussian random vectors decomposed into fixed and random effects:

$$
\Theta_{i}=\boldsymbol{\lambda}+c_{i} \beta+\boldsymbol{\eta}_{i}, \boldsymbol{\eta}_{i} \sim \mathcal{N}(0, \Omega), \quad \forall i=1, \ldots, r,
$$

where $\boldsymbol{\lambda}$ is an unknown vector of length $d$, called reference population parameter. $\beta$ is an unknown vector of length $p$ of covariate parameters and $c_{i}$ is a covariate matrix of size $d \times p$ given by the user. Examples of covariates used herein are presented in Section 3.3. Fixed effects are gathered in $(\boldsymbol{\lambda}, \beta)$. $\boldsymbol{\eta}_{i}$ denotes a Gaussian vector of random effects, with covariance matrix $\Omega$. $\left(e_{i j}\right)$ and $\left(\boldsymbol{\eta}_{i}\right)$ are assumed to be mutually independent. If the $l$-th parameter component $\theta_{i l}$ of $\boldsymbol{\Theta}_{i}$ is known to be positive, e.g. $x_{0}, \tau, T$ in equation (3), a $\log$ parametrization is used such that $\log \left(\theta_{i l}\right)$ is a Gaussian variable. Model 
hyper-parameters to be estimated from experimental data are gathered in

$$
\boldsymbol{\psi}=(\boldsymbol{\lambda}, \beta, \Omega, \sigma)
$$

To simplify the presentation of the results given in Section 4, a positive value of any fixed effect in $\beta$ will denote a local improvement of the therapeutic response due to the covariate $c_{i}$ and conversely a negative value will be synonymous of therapeutic degradation. Thereafter, the notation $\beta_{\theta, c}$ will be used to denote the effect of the covariate $c$ on the model parameter $\theta$.

\section{Materials and Methods}

\subsection{Statistical methods}

\subsubsection{Parameter estimation}

The parameter estimation of non-linear mixed models is complex: the likelihood has no explicit form because of the nonlinearity of the regression function in the individual parameters. The Expectation-Maximization (EM) algorithm is a generalization of the maximum likelihood estimation to the non-observed or incomplete data case ${ }^{23,24}$. For non-linear mixed models, the non-observed (or hidden) data are the individual parameter vector $\boldsymbol{\Theta}=\left(\boldsymbol{\Theta}_{1}, \ldots, \boldsymbol{\Theta}_{r}\right)$, the complete data are the $(\boldsymbol{y}, \boldsymbol{\Theta})$. Starting with an initial value $\widehat{\boldsymbol{\psi}}_{0}$ of the model hyperparameters defined in (13), the EM algorithm seeks to find the maximum likelihood estimate by iteratively applying the following two steps:

(1) Expectation step (E-step): calculation of the expected value of the $\log$ likelihood function, with respect to the conditional distribution of $\Theta$ given $\boldsymbol{y}$ under the current estimate of the parameters $\widehat{\boldsymbol{\psi}}_{m}$ at the $m$-th 
iteration

$$
Q\left(\boldsymbol{\psi} \mid \widehat{\boldsymbol{\psi}}_{m}\right)=E\left(L_{c}(\boldsymbol{y}, \boldsymbol{\Theta} ; \boldsymbol{\psi}) \mid \boldsymbol{y} ; \widehat{\boldsymbol{\psi}}_{m}\right)
$$

where $L_{c}(\boldsymbol{y}, \boldsymbol{\Theta} ; \boldsymbol{\psi})$ is the log-likelihood of the complete data.

(2) Maximization step (M-step): update of $\widehat{\boldsymbol{\psi}}_{m}$ by $\widehat{\boldsymbol{\psi}}_{m+1}$ by maximizing this quantity

$$
\widehat{\boldsymbol{\psi}}_{m+1}=\arg \max Q\left(\boldsymbol{\psi} \mid \widehat{\boldsymbol{\psi}}_{m}\right)
$$

For cases in which the E-step has no analytic form, Delyon et al. introduced in $^{25}$ a stochastic version of the EM algorithm that estimates the integral

$Q\left(\boldsymbol{\psi} \mid \widehat{\boldsymbol{\psi}}_{m}\right)$ by a stochastic approximation procedure via the simulation of the individual parameters $\boldsymbol{\Theta}$ under the posterior distribution $p(\boldsymbol{\Theta} \mid \boldsymbol{y} ; \boldsymbol{\psi})$. For nonlinear mixed models, the simulation step is not direct. Kuhn and Lavielle proposed to use a Monte Carlo Markov Chain to simulate $\Theta^{22}$. They proved the convergence of the algorithm under general hypothesis. This algorithm is implemented in the Monolix software (http://www.monolix.org/).

\subsubsection{Hypothesis testing and model selection}

The estimation of the mixed model parameters is based on two main steps.

(1) The covariance matrix $\Omega$ (full or diagonal) and the output error model $\epsilon_{i j}$ (homoscedastic or heteroscedastic) are selected in a first step. This double selection is carried out by implementing a full $2^{2}$ factorial design composed of 2 two-level factors and four combinations: (full-homo;fullhete;diag-homo;diag-hete). Two classic information criteria: AIC (Akaike's Information Criterion) and BIC (Bayesian Information Criterion) ${ }^{26,27}$ 
are used as selection statistics. The selected covariance matrix and error model are the ones that minimize AIC and BIC. The latter criteria require the computation of the model log-likelihood. This log-likelihood, which has no analytical form, is estimated using a Monte-Carlo Importance Sampling algorithm.

(2) In a second step, a Likelihood Ratio Test (LRT) is used to select covariates $\beta$. If the LRT is not significant with a significance level of $5 \%$, the effect of the covariate is removed.

\subsection{Experimental setup of data}

Female nude mice were used for tumor implantation. Female athymic Foxn1 nude mice $(\mathrm{nu} / \mathrm{nu})$ were obtained from Harlan (Gannat, France), and used at an age of 7-9 weeks and a weight of 20-25 g. Animal procedures were performed according to institutional and national guidelines. The tumor, a model of human malignant glioma (U87 cancer cell line), was maintained in vivo by sequential passages in nude mice. For the experiments, source tumors were excised, cleaned from necrotic tissue, cut into small chunks, and transplanted subcutaneously in the hind leg of each mouse. Three loco-regional therapies for cancer treatment were carried out: RT (radiotherapy), RCT (concomitant radiochemotherapy) and PDT (photodynamic therapy).

A group of 54 mice did not receive any treatment and was considered as the control group.

Radiotherapy was applied during 6 weeks to a group of 7 mice with a total dose of $40 G y$ per mouse for ionizing radiation. Concomitant radiochemotherapy was delivered during 1,2, 4 or 6 weeks to groups of 7 mice. RCT was based on the combination of topotecan (daily intraperitoneal injection, 5 days/week) and 
ionizing radiation (5 days/week). Total doses were $3 \mathrm{mg} / \mathrm{kg}$ for topotecan and 40Gy for ionizing radiation. RT and RCT treatments started when tumors reached a mean diameter of $8 \pm 1 \mathrm{~mm}$.

For the PDT group, tumors were treated when they reached a size of $5 \pm 1 \mathrm{~mm}$ mean diameter. A new targeted photosensitizing agent, a chlorin conjugated to heptapeptide targeting neuropilin-1, was used ${ }^{28}$. The in vivo treatment condition was: drug-light interval: $4 \mathrm{hrs}$, agent dose: $2.80 \mathrm{mg} / \mathrm{kg}$, fluence: $120 \mathrm{~J} / \mathrm{cm}^{2}$ and fluence rate: $85 \mathrm{~mW} / \mathrm{cm}^{2}$. The PDT group was composed of 8 mice.

The complete biological and medical protocols are defined in ${ }^{29,30}$. For each subject, the observation period started at the beginning of treatment $(t=0)$. Measurements were then carried out until the tumors reached a size of $15 \mathrm{~mm}$ in diameter, the legal barrier at which time the mice were sacrificed by cervical dislocation.

\subsection{Comparative studies in oncology}

Three cases were examined in this study:

- Treated and not-treated (control) tumors. Results of non-treated tumor growth identification are presented in Section 4.1.

- Three therapeutic strategies for cancer treatment (RT, RCT, PDT). Treatments RCT and PDT were encoded by binary covariates taking value 1 when the therapy was applied and 0 otherwise, RT being the reference treatment. Results are presented in Section 4.2.

- Four different treatment durations for the concomitant radiochemotherapy: 1, 2, 4, 6 weeks for constant total doses of drug and radiation. This treatment duration was described by a categorical covariate discretized into four levels: 
$\{1,2,4,6\}$. Results are presented in Section 4.3.

\section{Results}

The results of the non-treated tumor growth are presented first. Then the comparison of the three treatments is detailed. Finally, the effect of the treatment duration in concomitant radiochemotherapy is identified.

\subsection{Non-treated tumor growth identification}

The model selection procedure, presented in Section 3.1.2 and based on the minimization of AIC and BIC, applied to the natural growth responses of U87-tumors $(u=0)$ has led to opting for the homoscedastic error model and a diagonal covariance matrix $\Omega(\mathrm{AIC}=1413, \mathrm{BIC}=1423)$.

Estimates of the model parameters are given in Table 2. In Figure 2, the empirical distributions of the equation residuals $(e)$ and their quantile-quantile plot confirm the Gaussian assumption stated in (10). A comparison of predicted responses with observations for a few subjects of the U87 control group is displayed in Figure 3(a). The linear trend of the diameter growth is manifest. In other terms, the linearity assumption about the natural tumor growth is corroborated by the present results.

4.2 Treated tumor growth identification and comparison of the three locoregional therapies

In vivo data of three loco-regional therapies - radiotherapy (RT), concomitant radiochemotherapy $(\mathrm{RCT})$ and photodynamic therapy (PDT)- were an- 
alyzed. In the model selection procedure, AIC and BIC criteria were minimal for homoscedastic error model $\left(\epsilon_{i j}\right)$ and a diagonal covariance matrix $\Omega$ with $\Omega=\operatorname{diag}\left(\omega_{x_{0}}, \omega_{T}, \omega_{k_{1}}, \omega_{\tau}, \omega_{k_{2}}, \omega_{k_{3}}\right)$. Then the three treatment effects were compared with each other. The selection of the influent covariates, among RCT and PDT, on $\left(x_{0}, k_{1}, k_{2}, T, \tau, k_{3}\right)$ was then applied using the method detailed in Section 3.1.2. The final model $(\mathrm{AIC}=939)$ included five significant covariate effects: four effects due to PDT $\left(\beta_{x_{0}, P D T}, \beta_{k_{1}, P D T}, \beta_{k_{2}, P D T}, \beta_{k_{3}, P D T}\right)$ on parameters $x_{0}, k_{1}, k_{2}, k_{3}$ respectively and one effect induced by $\mathrm{RCT}, \beta_{\tau, R C T}$, on the time delay $\tau$. Parameter values are presented in Table 3. $\beta_{x_{0}, P D T}=-2.73 \mathrm{~mm}$, is particular and should not be compared with the other fixed effects. Indeed, it represents the significant variations of the tumor initial size $x_{0}$ between groups of mice, which are about $3 \mathrm{~mm}$ in this study case (see Experimental setup of data in Section 3.2).

The results emphasize that the three therapies lead to reducing the growth rate of tumors during the first phase in comparison with natural growth responses $\left(k_{1}<a\right.$ where the natural growth rate $a$ is given in Table 2$)$. The positive value of $\beta_{k_{1}, P D T}$ reveals an improved therapeutic efficiency (growth rate reduction) of PDT compared to RT and RCT during this phase. Conversely, RCT produces better therapeutical effects than PDT during the second phase as illustrated in Figure 3(c). Indeed, RT and RCT cause a transient decrease of the tumor diameter $\left(k_{2}>0\right)$ during the second phase while PDT leads to an opposite effect $\left(\beta_{k_{2}, P D T}<0\right)$, i.e. a momentary increase of tumor size over the same period of time. The RCT treatment also reveals a positive effect $\beta_{\tau, R C T}$ on the time delay $\tau$, meaning that RCT significantly defers tumor growth compared to RT and PDT. At steady-state of growth (late effect), the positive effect of PDT on the decrease rate $k_{3}$ of the tumor diameter indicates that PDT better limits the growth rate than RT and RCT during that period of time. These 
different previous effects can be observed in the experimental and predicted growth responses for a few mice treated by the three therapies, see Figure 3. Table 4 displays the correlation matrix of the parameter estimates, whose the content confirms the independence assumption between the model parameters.

These results emphasize the practical interest of such a model-based approach to characterize, analyze and compare anti-cancer therapeutic responses by using model parameters as therapeutic outcome indicators.

\subsection{Evaluation of the treatment-duration effect for the concomitant radioche- motherapy}

The last question was to estimate the effect of the treatment duration on growth of tumors treated by concomitant radiochemotherapy. The model selection strategy was applied as described in Section 3.1.2, a homoscedastic error model and a diagonal covariance matrix were selected. The final model only includes covariates on parameters $k_{2}$ and $\tau: \beta_{k_{2}, 2}, \beta_{\tau, 4}, \beta_{\tau, 6}$. The second indices 2, 4, 6 of the covariate effects denote values of the treatment duration. Parameter values are presented in Table 5 and Table 6 displays the correlation matrix of the parameter estimates. As previously, the estimated correlation coefficients confirm the independence assumption between the model parameters. The positive value of $k_{2}$ confirms the transient decrease of the tumor diameter in the second phase. The 2-week treatment duration increases the positive effect, $\beta_{k_{2}, 2}$, on this transient decrease while there is no significant effect for the 4- and 6-week treatment durations. The treatment duration has also a significant effect on $\tau$, estimated by $\beta_{\tau, 4}$ and $\beta_{\tau, 6}$. The longer the treatment, the more the tumor growth is delayed. Therefore, those two indicators allow the biologist to select the suited treatment modalities in order to opti- 
mize the therapeutic response. In this case, adding the total dose of radiation $D$ to the factors of the experimental design could bring new insight into the therapeutic effects of the treatment.

\section{Conclusion}

This paper proposes a new mixed effects model for diameter growth of treated and non-treated tumors. Compared to classic tumor volume models, its main advantage is the simplicity of its kinetic structure. Model parameters, composed of growth rates, growth delays or time constants are meaningful. They characterize the early, late and steady-state effects of anti-cancer treatments. In vivo results confirmed the relevance of the suggested model to describe the tumor growth responses to three loco-regional anti-cancer treatments. The main advantage of the mixed effects models is to introduce treatment factors into the model, e.g. the treatment duration, in order to estimate their influence on tumor growth while taking into account the inter-individual variability of in vivo growth responses. The presented results highlight the potential role of the parameter estimates as therapeutic outcome indicators. The application of the Linear-Exponential-Linear model structure to other cancer cell lines is currently in progress. A simplified version of this model, reduced to the steadystate phase of growth, was recently used to optimize treatment modalities of $\mathrm{PDT}^{31}$. Indeed, the main application of such a model is the optimization of anti-cancer treatment modalities by its combined use with optimal experiment designs, e.g. as proposed by Retout et al. in ${ }^{32}$ for mixed models optimal design. In this perspective, all potential factors of treatment have to be considered in the experimental design. 


\section{References}

1 Wiener N. Cybernetics or Control and Communication in the Animal and the Machine. MIT Press; 1948.

2 von Bertalanffy L. Principles and theory of growth. In: Fundamental Aspects of Normal and Malignant Growth. Nowinsky, W. W. ed. Amsterdam: Elsevier; 1960. p. 137.

3 Houghton PJ, Morton CL, Tucker C, Payne D, Favours E, Cole C, et al. The Pediatric Preclinical Testing Program: Description of Models and Early Testing Results. Pediatr Blood Cancer. 2007;49:928-940.

4 Fischer JJ. Mathematical simulation of radiation theory of solid tumors. I. Calculations. Acta Radiol Ther Phys Biol. 1971;10:73.

5 Cox EB, Woodburry MA, Meyers LE. A new model for tumor growth analysis based on a postulated inhibitory substance. Comp Biomed Res. 1980;13:437.

6 Swan GW. Tumor Growth Models and Cancer Chemotherapy. In: Thompson JR, Brown BW, editors. Cancer Modeling. Dekker; 1987. p. 91-179.

7 Hahnfeldt P, Panigrahy D, Folkman J, Hlatky L. Tumor Development under Angiogenic Signaling: A Dynamical Theory of Tumor Growth, Treatment Response, and Postvascular Dormancy. Cancer Research. 1999;59:4770-4775.

8 Sachs RK, Hlatky LR, Hahnfeldt P. Simple ODE Models of Tumor Growth and Anti-Angiogenic or Radiation Treatment. Mathematical and Computer Modelling. 2001;33:1297-1305.

9 Mandonnet E, Delattre JY, Tanguy ML, Swanson KR, Carpentier AF, Duffau H, et al. Continuous Growth of Mean Tumor Diameter in a Subset of Grade II Gliomas. Annals of Neurology. 2003;53(4):524-528.

10 Guiot C, Degiorgis PG, Delsanto PP, Gabriele P, Deisboeck TS. Does 
tumor growth follow a "universal law" ? J Theor Biol. 2004;229(3):289.

11 Tee D, DiStefano III J. Simulation of tumor-induced angiogenesis and its response to anti-angiogenic drug treatment: mode of drug delivery and clearance rate dependencies. Journal of Cancer Research and Clinical Oncology. 2004;130(1):15-24.

12 Ribba B, Saut O, Colin T, Bresch D, Grenier E, Boissel JP. A multiscale mathematical model of avascular tumor growth to investigate the therapeutic benefit of anti-invasive agents. J Theor Biol. 2006;243:532-541.

13 de Pillis LG, Gu W, Fister KR, Head T, Maples K, Murugan A, et al. Chemotherapy for tumors: an analysis of the dynamics and a study of quadratic and linear optimal controls. Mathematical Biosciences. 2007;209:292-315.

14 Dua P, Duab V, Pistikopoulos EN. Optimal delivery of chemotherapeutic agents in cancer. Computers and Chemical Engineering. 2008;32:99-107.

15 Newman WI, Lazareff JA. A mathematical model for self-limiting brain tumors. Journal of Theoretical Biology. 2003;222:361-371.

16 Drasdo D, Hoehme S. Individual-based approaches to birth and death in avascular tumors. Math and Comp Modelling. 2003;37:1163-1175.

17 Drasdo D, Höhme S. A single-cell-based model of tumor growth in vitro: monolayers and spheroids. Phys Biol. 2005;2:133-147.

18 Galle J, Aust G, Schaller G, Beyer T, Drasdo D. Individual cell-based models of the spatio-temporal organisation of multicellular systems - achievements and limitations. Cytometry, Cytometry A. 2006;69A:704-710.

19 Lindstrom M, Bates D. Nonlinear mixed-effects models for repeated measures data. Biometrics. 1990;46:673-687.

20 Samson A, Lavielle M, Mentré F. Extension of the SAEM algorithm to left-censored data in non-linear mixed-effects model: application to HIV dynamics model. Computational Statistics and Data Analysis. 
$2006 ; 51(3): 1562-74$.

21 Samson A, Lavielle M, Mentré F. The SAEM algorithm for group comparison tests in longitudinal data analysis based on non-linear mixed-effects model. Statistics in Medicine. 2007;26(27):4860-4875.

22 Kuhn E, Lavielle M. Maximum likelihood estimation in nonlinear mixed effects models. Computational Satistics and Data Analysis. 2005;49:10201028.

23 Dempster AP, Laird NM, Rubin DB. Maximum likelihood from incomplete data via the EM algorithm. Journal of the Royal Statistical Society, Series B (Methodological). 1977;39(1):1-38.

24 Mclachlan GJ, Krishnan T. The EM Algorithm And Extensions. John Wiley \& Sons; 2007.

25 Delyon B, Lavielle M, Moulines E. Convergence of a stochastic approximation version of the EM algorithm. Annals of Statistics. 1999;27:94-128.

26 Akaike H. A new look at the statistical model identification. IEEE Transactions on Automatic Control. 1974;19(6):716-723.

27 Schwarz G. Estimating the dimension of a model. Annals of Statistics. 1978;6(2):461-464.

28 Tirand L, Frochot C, Vanderesse R, Thomas N, Trinquet E, Pinel S, et al. A peptide competing with $\mathrm{VEGF}_{165}$ binding on neuropilin-1 mediates targeting of a chlorin-type photosensitizer and potentiates its photodynamic activity in human endothelial cells. Journal of Controlled Release. $2006 ; 111: 153-164$.

29 Pinel S, Chastagner P, Merlin JL, Marchal C, Taghian A, Barberi-Heyob M. Topotecan can compensate for protracted radiation treatment time effects in high grade glioma xenografts. J Neurooncol. 2006;76(1):31-8.

30 Tirand L, Thomas N, Dodeller M, Dumas D, Frochot C, Guillemin F, et al. Metabolic profile of a peptide-conjugated chlorin-type photosensitizer 
targeting neuropilin-1: an in vivo and in vitro study. Drug Metab Dispos. 2007;35:806-813.

31 Tirand L, Bastogne T, Bechet D, Linder M, Thomas N, Frochot C, et al. Response Surface Methodology: an extensive Potential to Optimize Photodynamic Therapy Conditions in vivo. To be published in International Journal of Radiation Oncology, Biology, Physics. 2009;

32 Retout S, Comets E, Samson A, Mentré F. Design in nonlinear mixed effects models: optimization using the Fedorov-Wynn algorithm and power of the Wald test for binary covariates. Stat Med. 2007;26(28):5162-79. 


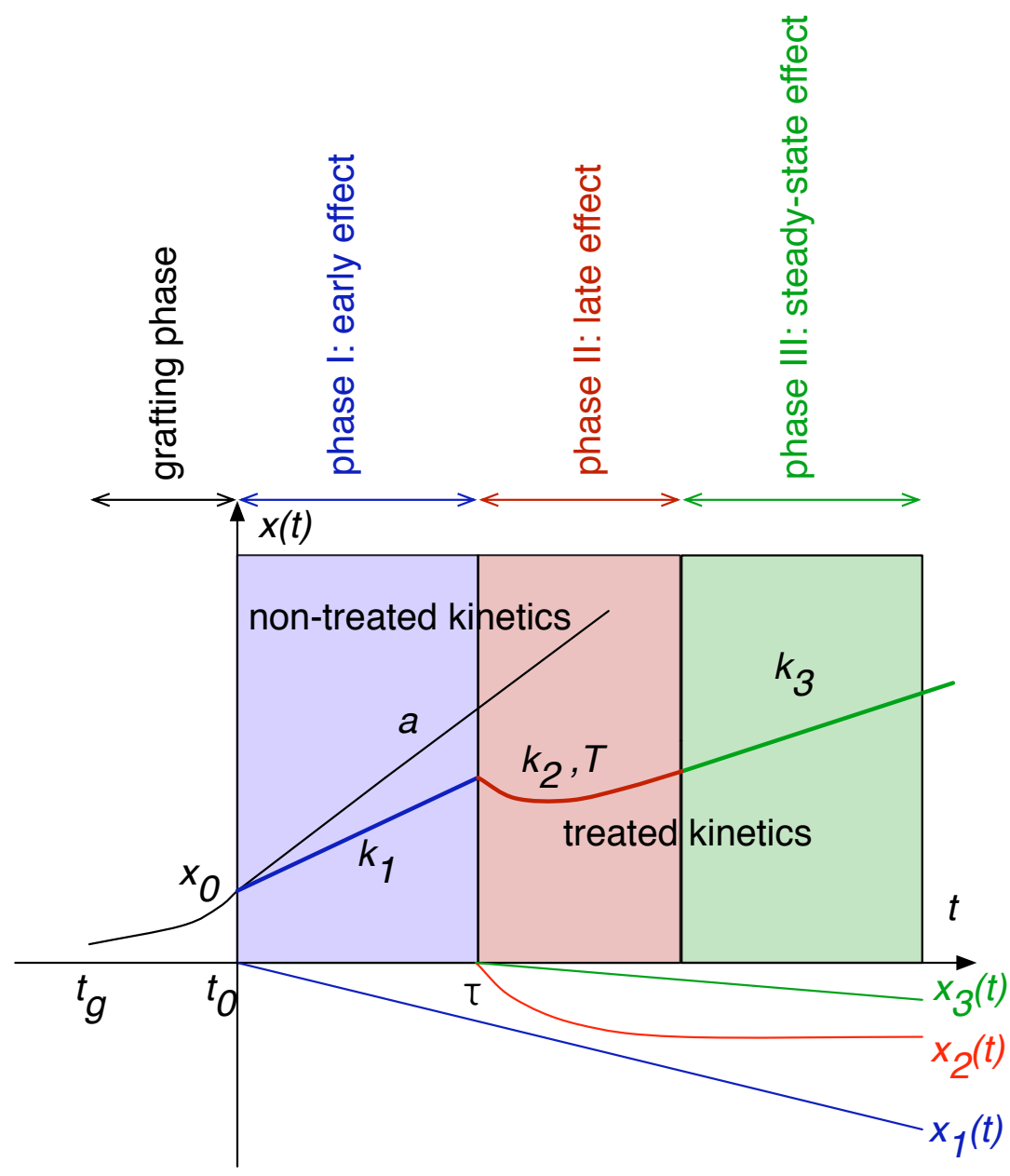

Figure 1. L- and LEL-model structures of the tumor diameter growth (LEL: linearexponential-linear). $t_{g}$ denotes the grafting time. 
Table 2

Parameter estimates and their standard errors (s.e.) for the non-treated tumor growth modeling. $\omega_{a}$ and $\omega_{x_{0}}$ are diagonal elements of the covariance matrix $\Omega$ defined in (12)

\begin{tabular}{lrr}
\hline param. & estimate & (s.e.) \\
\hline$x_{0}[\mathrm{~mm}]$ & 5.95 & $(0.22)$ \\
$a\left[d^{-1} y^{-1}\right]$ & 0.0604 & $(0.0025)$ \\
$\omega_{a}$ & 0.0166 & $(0.01)$ \\
$\omega_{x_{0}}$ & 0.264 & $(0.01)$ \\
$\sigma$ & 0.558 & $(0.018)$ \\
\hline
\end{tabular}




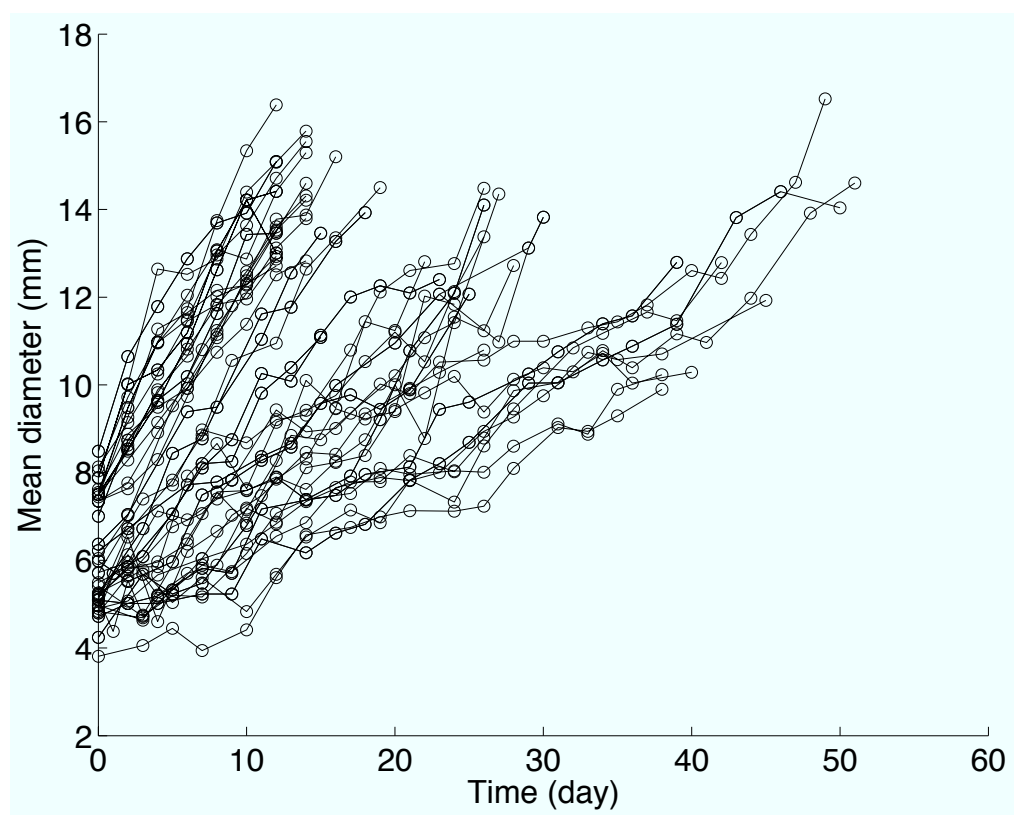

(a) Longitudinal data set of the control group

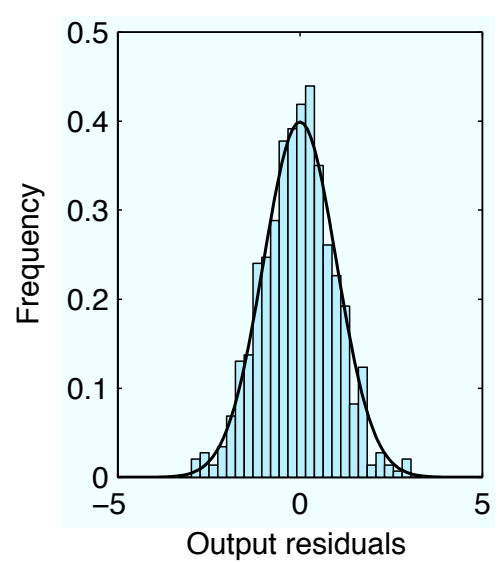

(b) Residual histogram

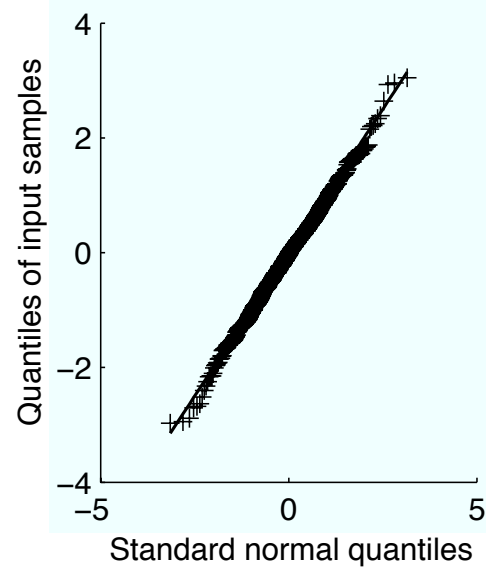

(c) Residual Q-Q plot

Figure 2. Longitudinal data set and residual analysis for the U87 control group 
Table 3

Parameter estimates, standard errors (s.e.) and p-values of the Wald test of the LEL-model parameters for three loco-regional therapies: RT, RCT and PDT (radiotherapy, concomitant radiochemotherapy and photodynamic therapy). Estimates of $\left(x_{0}, k_{1}, k_{2}, T, \tau, k_{3}\right)$ are values of $\boldsymbol{\lambda}$ (see Eq. 12) determined by the SAEM algorithm. Only significant covariate effects are presented.

\begin{tabular}{lrrlrrr}
\hline \hline parameter & estimate & (s.e.) & parameter & estimate & (s.e.) & p-value \\
\hline$x_{0}[\mathrm{~mm}]$ & 7.9 & $(0.12)$ & $\beta_{x_{0}, P D T}$ & -2.73 & $(0.2)$ & $<10^{-10}$ \\
$k_{1}\left[\mathrm{day}^{-1}\right]$ & -0.0394 & $(0.005)$ & $\beta_{k_{1}, P D T}$ & 0.0451 & $(0.0096)$ & $2.7 e-6$ \\
$k_{2}\left[\mathrm{day}^{-1}\right]$ & 0.0387 & $(0.015)$ & $\beta_{k_{2}, P D T}$ & -0.0633 & $(0.025)$ & 0.011 \\
$T[\mathrm{day}]$ & 8.54 & $(2.7)$ & & & & \\
$\tau[\mathrm{day}]$ & 10.3 & $(1.2)$ & $\beta_{\tau, R C T}$ & 6.53 & $(2.1)$ & 0.0021 \\
$k_{3}\left[\mathrm{day}{ }^{-1}\right]$ & -0.015 & $(0.0096)$ & $\beta_{k_{3}, P D T}$ & 0.0642 & $(0.016)$ & $5.2 \mathrm{e}-05$ \\
\hline$\omega_{x_{0}}$ & 0.317 & $(0.092)$ & $\omega_{T}$ & 3.02 & $(4.2)$ & \\
$\omega_{k_{1}}$ & 0.0177 & $(0.0032)$ & $\omega_{\tau}$ & 4.2 & $(0.74)$ & \\
$\omega_{k_{2}}$ & 0.0449 & $(0.0094)$ & $\omega_{k_{3}}$ & 0.0297 & $(0.0056)$ & \\
$\sigma$ & 0.484 & $(0.019)$ & & & & \\
\hline \hline
\end{tabular}

Table 4

Correlation matrix of the estimates for three loco-regional therapies: RT, RCT and PDT (radiotherapy, concomitant radiochemotherapy and photodynamic therapy).

\begin{tabular}{|c|c|c|c|c|c|c|c|c|c|c|c|}
\hline & $x_{0}$ & $\beta_{x_{0}, P D T}$ & $k_{1}$ & $\beta_{k_{1}, P D T}$ & $k_{2}$ & $\beta_{k_{2}, P D T}$ & $T$ & $\tau$ & $\beta_{\tau, R C T}$ & $k_{3}$ & $\beta_{k_{3}, P D T}$ \\
\hline$x_{0}$ & 1 & & & & & & & & & & \\
\hline$\beta_{x_{0}, P D T}$ & -0.58 & 1 & & & & & & & & & \\
\hline$k_{1}$ & -0.22 & 0.14 & 1 & & & & & & & & \\
\hline$\beta_{k_{1}, P D T}$ & -0.31 & 0.12 & -0.54 & 1 & & & & & & & \\
\hline$k_{2}$ & -0.06 & 0.03 & 0.04 & -0.01 & 1 & & & & & & \\
\hline$\beta_{k_{2}, P D T}$ & 0.03 & 0 & -0.02 & 0.01 & -0.6 & 1 & & & & & \\
\hline$T$ & -0.01 & 0.01 & 0.01 & -0.02 & -0.08 & 0.13 & 1 & & & & \\
\hline$\tau$ & 0.02 & -0.05 & -0.03 & 0.11 & 0.07 & -0.13 & -0.07 & 1 & & & \\
\hline$\beta_{\tau, R C T}$ & 0.03 & 0 & -0.02 & -0.04 & -0.01 & 0.05 & -0.05 & -0.56 & 1 & & \\
\hline$k_{3}$ & 0.09 & -0.05 & -0.06 & 0.03 & 0.17 & -0.08 & 0.31 & 0.03 & -0.01 & 1 & \\
\hline$\beta_{k_{3}, P D T}$ & -0.05 & 0.14 & 0.04 & -0.17 & -0.1 & 0.14 & -0.27 & -0.09 & 0.06 & -0.63 & 1 \\
\hline
\end{tabular}


Table 5

Parameter estimates, standard errors (s.e.) and p-values of the Wald test of the LEL-model parameters for three concomitant radiochemotherapy. Estimates of $\left(x_{0}, k_{1}, k_{2}, T, \tau, k_{3}\right)$ are values of $\boldsymbol{\lambda}$ (see Eq. 12) determined by the SAEM algorithm. Only significant covariate effects are presented. Indices $2,4,6$ of the covariate effects denote values of the treatment duration (see Section 3.3).

\begin{tabular}{lrrlrrr}
\hline \hline parameter & estimate & (s.e.) & parameter & estimate & (s.e. $)$ & p-value \\
\hline$x_{0}[\mathrm{~mm}]$ & 8.04 & $(0.13)$ & & & & \\
$k_{1}\left[d a y^{-1}\right]$ & -0.0171 & $(0.0014)$ & & & & \\
$k_{2}\left[d a y^{-1}\right]$ & 0.0438 & $(0.0044)$ & $\beta_{k_{2}, 2}$ & 0.0203 & $(0.0093)$ & 0.029 \\
$T[d a y]$ & 10.8 & $(2.1)$ & & & & \\
$\tau[d a y]$ & 14.7 & $(0.64)$ & $\beta_{\tau, 4}$ & 3.92 & $(1)$ & 0.00016 \\
& & & $\beta_{\tau, 6}$ & 7.06 & $(1)$ & $1.1 \mathrm{e}-11$ \\
$k_{3}\left[d a y^{-1}\right]$ & 0.00762 & $(0.0032)$ & & & & \\
\hline$\omega_{x_{0}}$ & 0.6 & $(0.094)$ & $\omega_{T}$ & 2.07 & $(3)$ & \\
$\omega_{k_{1}}$ & 0.0061 & $(0.0011)$ & $\omega_{\tau}$ & 1.6 & $(0.4)$ & \\
$\omega_{k_{2}}$ & 0.0169 & $(0.0032)$ & $\omega_{k_{3}}$ & 0.00987 & $(0.0019)$ & \\
$\sigma$ & 0.449 & $(0.013)$ & & & & \\
\hline
\end{tabular}


Table 6

Correlation matrix of the estimates for three modalities of concomitant radiochemotherapy.

\begin{tabular}{r|ccccccccc|} 
& $x_{0}$ & $k_{1}$ & $k_{2}$ & $\beta_{k_{2}, 2}$ & $T$ & $\tau$ & $\beta_{\tau, 4}$ & $\beta_{\tau, 6}$ & $k_{3}$ \\
\hline$x_{0}$ & 1 & & & & & & & & \\
$k_{1}$ & -0.2 & 1 & & & & & & & \\
$k_{2}$ & -0.07 & 0.12 & 1 & & & & & & \\
$\beta_{k_{2}, 2}$ & -0.01 & 0.02 & -0.47 & 1 & & & & & \\
$T$ & 0 & 0.01 & 0.19 & -0.11 & 1 & & & & \\
$\tau$ & 0.05 & -0.15 & -0.03 & 0.06 & -0.28 & 1 & & & \\
$\beta_{\tau, 4}$ & 0 & 0.03 & 0.06 & -0.05 & 0.03 & -0.58 & 1 & & \\
$\beta_{\tau, 6}$ & -0.01 & 0.04 & 0.05 & -0.05 & 0.04 & -0.61 & 0.39 & 1 & \\
$k_{3}$ & 0.05 & -0.08 & 0.27 & -0.11 & 0.83 & -0.15 & 0.02 & 0.02 & 1 \\
\hline
\end{tabular}



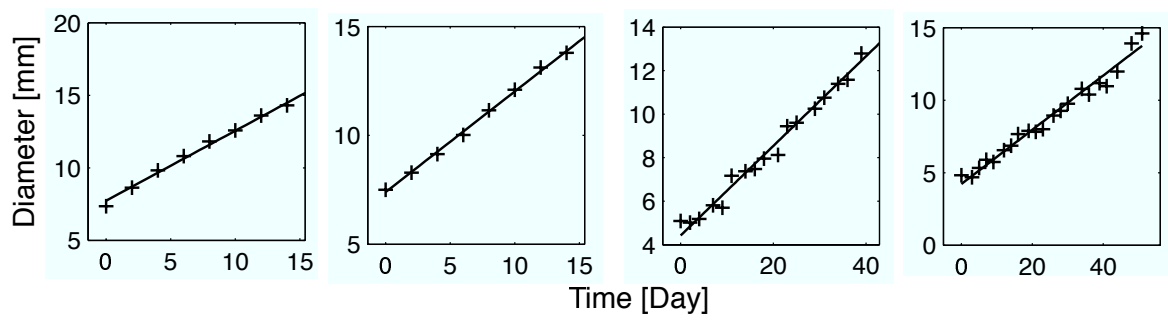

(a) Control responses
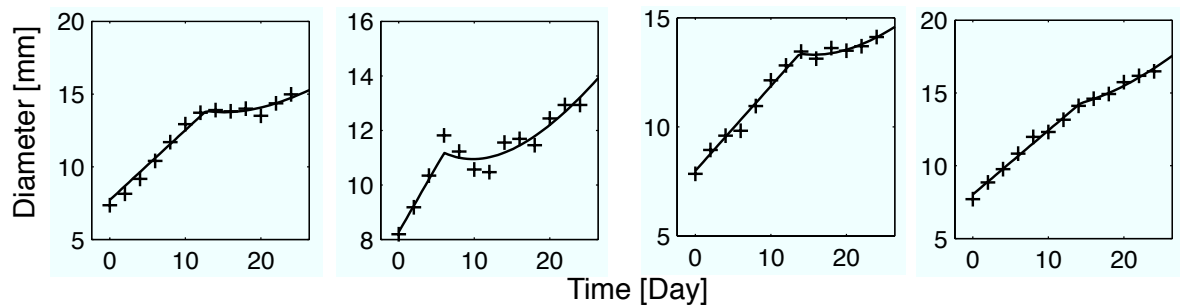

(b) Radiotherapy responses
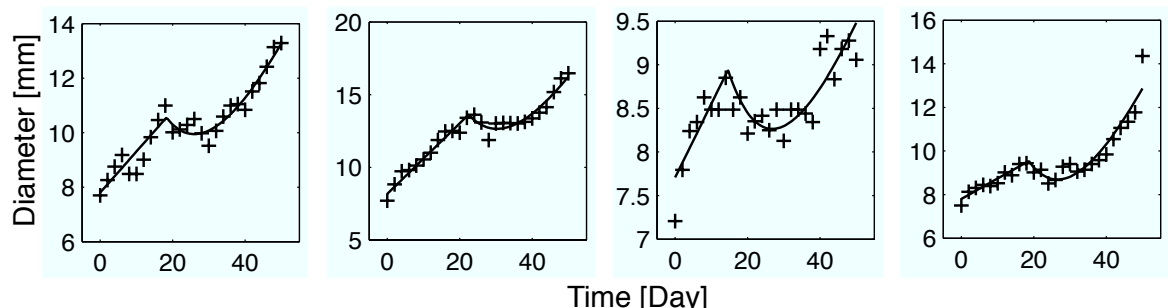

(c) Concomitant radiochemotherapy responses
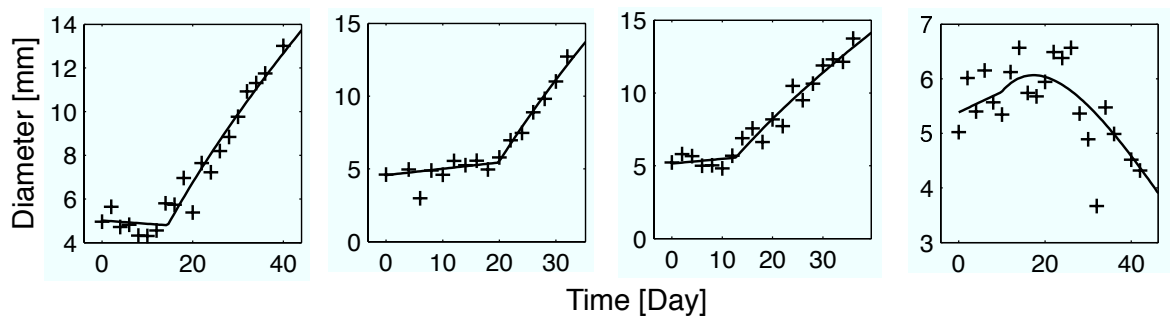

(d) Photodynamic therapy responses

Figure 3. Diameter growth kinetics of U87 tumors and predicted responses. Four growth kinetics are shown for each study case. The first row (a) exhibits the linear growth of the tumor diameter for the control (non-treated) group. The second row (b) displays the therapeutical responses of the radiotherapy where the early and late effects of the treatment clearly appear. The responses of the concomitant radiochemotherapy are presented in the third row (c). They express a larger late effect, characterized by a deeper decrease of the tumor diameters, than for the radiotherapy treatment. The last row (d) shows the photodynamic therapy responses. They particularly point out an improved early effect of the treatment and more precisely a significant reduction of the growth rate during the first phase. A total cure is observed in the last figure. In all cases, the predicted responses provided by the model are close to the observed growth kinetics, whatever the anticancer treatment used. 